the patients fasting periphery venous flood acquired on the second morning, fasting plasma glucose (FPG), triglyeride (TG), low density lipoprotein-cholesterol (LDL-C), high density lipoprotein-cholesterol (HDL-C), creatinine (Cre) are tested by auto-biochemistry instrument, enzyme linked immunosorbent assay adopted. 3. Twodimensional and Doppler echocardiography was performed using a Vivid 7 Dimension echo machine. Left ventricle end-diastolic diameter (LVEDD) and left atrial (LA) dimensions were standard $M$-mode measurements. Left ventricle ejection fraction (LVEF) was calculated using the modified Simpson's rule. 4. After centrifugation, $0.5 \mathrm{ml}$ clear supernatant liquid of fasting periphery venous flood deserved in $-80^{\circ} \mathrm{C}$ less than 5 months. APN concentration was measured by radio-immunity method. 5 . Record each general state, including BMI, medicine taking, whether with or without hypertension, coronary heart disease and so on. All data are expressed as mean \pm SD. All analyses were performed using SPSS 17.0.

Results Adiponectin concentration in persistent AF was significantly higher than in control group and paroxymal group. Covariance analysis revealed that plasma adiponectin was also significantly associated with the presence of arterial fibrillation $(p<0.05)$. Univariance analysis TG has influence on APN. Multiple linear regression show APN correlated negatively with TG, and APN in persistent AF group is higher than paroxymal AF group and controls. Conclusion High plasma adiponectin levels are associated with the presence of persistent and permanent AF. Adiponectin concentration was correlated negatively with TG.

\section{e0677 INCREASED PLASMA NTERMINAL PROBTYPE NATRIURETIC PEPTIDE IN PATIENTS WITH HEPATITIS C VIRUS INFECTION}

doi:10.1136/hrt.2010.208967.677

\begin{abstract}
1,2 Wenliang Che, ${ }^{3}$ Wenling Liu, ${ }^{1}$ Yidong Wei, ${ }^{1}$ Yawei Xu, ${ }^{4}$ Yisen Chen, ${ }^{4}$ Yunli Huang, ${ }^{3}$ Lei Li, ${ }^{5}$ Jie Xiao, ${ }^{6}$ Akira Matsumori, ${ }^{2,3}$ Dayi Hu. ${ }^{1}$ Shanghai Tenth People's Hospital, Tongji University, Shanghai, China; ${ }^{2}$ Heart, Lung and Blood Vessel Center, Tongji University, Shanghai, China; ${ }^{3}$ Heart Center, Peking University People's Hospital, Beijing, China; ${ }^{4}$ Beijing YouAn Hospital, Capital Medical University, Beijing, China; ${ }^{5}$ Department of Cardiovascular Medicine, Beijing Tongren Hospital, Beijing, China; ${ }^{6}$ Department of Cardiovascular Medicine, Kyoto University Graduate School of Medicine, Kyoto, Japan
\end{abstract}

Objectives Some studies suggested a possible role for hepatitis C virus (HCV) in the pathogenesis of cardiovascular diseases (CVD). $\mathrm{N}$-terminal pro-brain natriuretic pepetide (NT-proBNP) has been proposed to be a neurohumoral marker of cardiovascular risk. Few prior studies have evaluated such levels in HCV infection. Accordingly, the objectives of the present study were to investigate circulating levels of NT-proBNP and their relevance in patients with $\mathrm{HCV}$ infection.

Methods We collected $131 \mathrm{HCV}$-infected patients and 131 age and gender matched healthy individuals from January 2006 to October 2007 in China. Demographics, clinical data were collected and circulating NT-proBNP was analysed, and 63 of patients were also consecutively evaluated with echocardiography.

Results The level of serum NT-proBNP was higher in HCV-infected patients compared with controls $(76.62 \mathrm{fmol} / \mathrm{ml}$ vs $51.83 \mathrm{fmol} / \mathrm{ml}$, $\mathrm{p}<0.001$, geometric means), even in HCV-infected patients without cardiovascular abnormalities (CVD history and /or abnormalities of ECG) NT-proBNP also increased $(63.46 \mathrm{fmol} / \mathrm{ml}$ vs $48.14 \mathrm{fmol} / \mathrm{ml}$, $\mathrm{p}=0.015$, geometric means). A NT-proBNP level in the highest tertile was associated with a higher risk of cardiovascular abnormalities, with OR of 17.91 (95\% CI, 3.71 to 86.47). MVE/MVA, LVEF and FS were significantly lower among patients in the highest NT-proBNP tertile, whereas MVA was higher. In addition, compared with normal values of healthy Chinese population $(39.35 \% \pm 4.26 \%)$, the value of FS $(36.76 \% \pm 5.50 \%, p=0.015)$ was lower in patients whose serum NT-proBNP level was higher than median of controls $(>56.17 \mathrm{fmol} / \mathrm{ml}, \mathrm{n}=37)$.
Conclusions HCV infected individuals had higher NT-proBNP levels than age matched controls, which show a possible cardiac functional evidence for a pathogenic link between HCV and CVD. The finding is consistent with an increased incidence of HCV or HCV antibody described in some CVD patients.

\section{e0678 PROTEIN AND MRNA EXPRESSION OF CX40 IN CRISTA TERMINALIS OF PATIENTS SUFFERED FROM RHEUMATIC HEART DISEASE WITH CHRONIC ATRIAL FIBRILLATION}

doi:10.1136/hrt.2010.208967.678

Zhao Feng, Zhou Yong, Li Li, Yao Jun-Di, Jiang Yi-Feng, Xu Zhi-Yun. Department of Cardiology, The 411th Hospital of PLA, Shanghai, China

Objective To investigate the protein expression and mRNA expression of $\mathrm{Cx} 40$ in crista terminalis of patients suffered from rheumatic heart disease with chronic atrial fibrillation. And evaluate the function of the remodelling of $\mathrm{Cx} 40$ in development and maintenance of atrial fibrillation in rheumatic heart disease.

Methods A small piece of myocardial specimen was acquired from crista terminalis during the operation in 20 patients who need operation therapy for rheumatic mitral valve disease and six patients undergoing other cardiac surgery served as control group. Western blot was used to detect expression of Cx40. Cx40 mRNA expression was detected by real-time fluorescence quantitative PCR method.

Results Compared with sinus rhythm, Cx40 expression was decreased in chronic atrial fibrillation. But, the difference of $\mathrm{Cx} 40$ mRNA expression among the three groups had no statistical significance.

Conclusion The remodelling of $\mathrm{Cx} 40$ plays an important role in the development and maintenance of atrial fibrillation in rheumatic heart disease. And the mechanism of the remodelling of Cx40 remains in the level after transcription of $\mathrm{Cx} 40$ gene.

\section{e0679 DEVELOPMENT OF A RAPID QUANTITATIVE DETECTION OF NT-PROBNP BASED ON SUPERPARAMAGNETIC NANOPARTICLES AS LABELS IN THE LATERAL FLOW IMMUNOASSAY}

doi:10.1136/hrt.2010.208967.679

${ }^{1}$ Wang Yanyan, ${ }^{2}$ Wei Meng, ${ }^{2}$ Li Jingbo, ${ }^{2}$ Zhu Wei, ${ }^{2}$ Xu Quanfu, ${ }^{1}$ Gu Shuiming. ${ }^{1}$ Shanghai Xuhui Central Hospital; ${ }^{2}$ Shanghai 6th Peoples Hospital Shanghai Jiaotong University School Of Medicine

Objective To establish a lateral flow immunoassay (LFIA) system for rapid, economic and quantitative detection of $\mathrm{N}$ terminal pro brain natriuretic peptide (NT-proBNP).

Method In this study, superparamagnetic nanoparticles (MNPs) were used as labels, the immuno-nanoparticles were prepared by coupling monoclone antibody specific to NT-proBNP onto MNPs, then the immunonanoparticles were used to prepare the conjugate pad of the magnetic LFIA of NT-proBNP. Another monoclone antibody specific to NT-proBNP (capture antibody) and secondary antispecies antibodies were immobilised at test line and control line, respectively. Then the magnetic LFIA for detection of NT-proBNP were established and applied to test standard samples of different NT-proBNP concentrations. The magnetic field produced by MNPs in the test line are measured by a high sensitive magnetic assay reader. From the linear relation between magnetic signal intensities and NT-proBNP concentrations, we can achieve quantitative detection of NT-proBNP. Some factors which may influence the detection sensitivity of this system were also studied, such as the amount of antibody immobilised in the Test line and the amount of antibody per MNP. 
Results The sensitivity of this magnetic LFIA of NT-proBNP was $0.01 \mathrm{ng} / \mathrm{ml}$, the detection range was reached five orders of magnitude, and the detection time was within $15 \mathrm{~min}$.

Conclusion It was showed that this magnetic LFIA of NT-proBNP has a high sensitivity, wide detection range and short detection time. It is a simple, rapid, accurate, quantitative and point-of-care testing which deserve to be spread and industrialised.

\section{e0680 RELATIONSHIP BETWEEN POLYMORPHISM OF ACE AND THE CURATIVE EFFECT OF METOPROLOL ON CHRONIC HEART FAILURE}

doi:10.1136/hrt.2010.208967.680

Miao Peizhi, Gu Shuiming. Shanghai Xuhui Central Hospital, Department of Cardiology, Shanghai, China

Objective To investigate the effect of metoprolol on chronic heart failure and to discuss the relationship between polymorphism of ACE and the curative effect of metoprolol.

Methods 118 patients with chronic heart failure were included randomly and were divided into two groups. In control group (group A), the patients were treated with benazapril 2.5-10 mg once daily and routinely, in treatment group the patients were treated additionally with metoprolol $12.5-100 \mathrm{mg}$ once daily. The period of treatment for all patients was 2 years. ACE polymorphism was detected by a PCR.

Results The patients encountered heart failure, myocardial infarction, malignant arrhythmia and sudden death in metoprolol group were lower than those of control group, the difference was significant $(p<0.05)$. PRA was significant increased and AngII, ALD were significant lowered in two groups after treatment. The frequency of DD genotype in metoprolol effective group was 0.47 and that in metoprolol ineffective group was 0.13 , the difference was significant $(p<0.05)$. The effective rate of DD genotype was $90.9 \%$, that of ID genotype was $76.9 \%$, that of II genotype was $54.2 \%$, and the effective rate of DD genotype was significantly higher than II genotype $(\mathrm{p}<0.05)$.

Conclusion Adding metoprolol to the treatment of chronic heart failure can improve heart function. The polymorphism of the ACE (I/D) is helpful for the diagnosis of the therapeutic efficacy of metoprolol in chronic heart failure.

\section{e0681 EFFECT OF CHEMOTATIC FACTOR FKN ON NF-KB AND TNF- $\alpha$ EXPRESSION IN PERIPHERAL BLOOD MONOCYTES AND THE ROLE OF PI3K}

doi:10.1136/hrt.2010.208967.681

Sun Jian, Guo Hui-jiao, Lei Ming-ming, Hou Wen-li, Yang Chun-yan, Wu Zhe. The First Hospital of Ji lin University, Chang chun, China

Objective In the present study, the effect of Fractalkine (FKN) on the expression of NF- $\mathrm{KB}$ and TNF- $\alpha$ induced by FKN was investigated one of possible signal transduction pathways of FKN/CX3CR1 in atherosclerosis, and the role of PI3K were also investigated.

Methods 1) Peripheral blood monocytes were isolated from fresh blood of healthy volunteers by Ficoll-Paque gradient centrifugation. 2) Divide the extractive peripheral blood monocytes into four groups :control group, FKN group, LY294002 group and PDTC group. 3) Measure the NF- $\mathrm{BB}$ expression of monocytes from each group by Western Blot. 4) Collect the supernatant of monocytes from each group, determine the expression of TNF- $\alpha$ by ELISA.

Result 1. The expression of NF-KBand TNF- $\alpha$ in FKN group was increased, compared with that control group $(p<0.05)$. 2 . The expression of NF- $\kappa$ B and TNF- $\alpha$ in LY294002 group was decreased, compared with that FKN group $(\mathrm{p}<0.05)$.
Conclusions FKN-CX3CR1 increase the expressions of NF- $\mathrm{KB}$ and TNF- $\alpha$ in peripheral blood monocytes, which may be one of the mechanisms of contributing to the progression of atherosclerosis; After interacting with its receptor CX3CR1, FKN activates the PI3K by coupled with G-protein and then initiates intracellular signal conductive mechanism.

\section{Q0682 THE COMBINED USE OF UROKINASE AND GLYCOPROTEIN IIB/IIIA-TARGETED MICROBUBBLES RECANALIZE RABBIT FEMORAL ARTERY WITH THROMBOTIC OCCLUSIONS}

doi:10.1136/hrt.2010.208967.682

Yu-ming Mu, Li-Na Guan, Ling Li, Chao-Feng Guo, Chun-Me Wang. Department of Echocardiography, Center of the Medical Ultrasound, The First Affiliated Hospital of Xinjiang Medical University

Objective To determine the effect of the combined use of urokinase and glycoprotein IIb/IIIa-targeted microbubbles prepared by direct conjugation method to dissolve the thromb.

Methods Urokinase and RGDS were in conjunction with microbubbles (SonoVue) by the direct conjugation method. The size, shape, fluorescent intensity, the binding rates of Urokinase and RGDS, and the activity of Urokinase were measured and analysed. A total of 42 rabbits with platelet-rich thrombi in the femoral artery were randomised into seven treatment groups ( $\mathrm{n}=6)$ : 1) ultrasound alone (US); 2) ultrasound plus non-targeted microbubbles (US+M); 3) urokinase alone (UK); 4) ultrasound, non-targeted microbubble and urokinase (US+M+UK); 5) ultrasound plus RGDS microbubble (US+R); 6) RGDS microbubble plus urokinase (R+UK); and 7) ultrasound, RGDS microbubble and urokinase (US+R+UK). US in diagnostic ultrasound were simultaneously applied over the thrombus up to $30 \mathrm{~min}$. The thrombolytic effect was evaluated at 120 min post treatment

Results SonoVue, Urokinase and RGDS were combined successfully. In vitro thrombolysis experiment indicated that the urokinase in the prepared contrast agent had activity $(p<0.01)$. For US, UK, US $+M$, $\mathrm{US}+\mathrm{R}$ and US $+\mathrm{M}+\mathrm{UK}$ groups, recanalisation was failed with the blood flow less than $15 \%$ of baseline. The blood flow for $\mathrm{R}+\mathrm{UK}$ was $15 \% \sim 49 \%$ of baseline, while that of US $+\mathrm{R}+\mathrm{UK}$ was more than $75 \%$ of baseline $(\mathrm{p}<0.001)$

Conclusion The combined use of urokinase and glycoprotein IIb/ IIIa-targeted microbubbles is effective in targeting thromb and recanalizing thrombolytic occlusion.

\section{e0683 CLINICAL SIGNIFICANCE OF AUTOANTIBODIES AGAINST CARDIAC TROPONIN I IN PATIENTS WITH MYOCARDIAL INFARCTION AND CHRONIC HEART FAILURE}

doi:10.1136/hrt.2010.208967.683

Yang Di, Bian Zhiping, Ji Peng, Li Bing, Chen Xiangjian, Xu Jindan, Gu Chunrong, Zhang Jinan. Institute of Cardiovascular Disease, First Affiliated Hospital of Nanjing Medical University, Nanjing

Objective Autoantibodies against cardiac troponin I (cTnI) have been described in the serum from patients with dialated cardiomyopathy and heart failure. The clinical significance of these autoantibodies remains unknown. The present study was designed to evaluate the relationship between the serum level of autoantibodies against cardiac troponin I and the prognosis of patients with myocardial infarction (MI) and chronic heart failure (CHF).

Methods 97 patients were studied in the present study, including 38 patients (68.3 \pm 7.9 years, 28 males) with $\mathrm{MI}$ and 59 patients (63.3 \pm 14.6 years, 44 males) with CHF. The patients were recruited in the First Affiliated Hospital of Nanjing Medical University from 2005 to 2008.78 healthy control subjects were enrolled in the study. The 